\title{
A BARREL IFR INSTRUMENTED WITH LIMITED STREAMER TUBES FOR BABAR EXPERIMENT
}

\author{
MIRCO ANDREOTTI \\ University/INFN of Ferrara \\ Via Saragat 1, \\ 44100, Ferrara, Italy \\ E-mail: mandreot@fe.infn.it
}

on behalf of the BaBar LST Collaboration

\begin{abstract}
The new barrel Instrumented Flux Return (IFR) of BABAR detector will be reported here. Limited Streamer Tubes (LSTs) have been chosen to replace the existing RPCs as active elements of the barrel IFR. The layout of the new detector will be discussed: in particular, a cell bigger than the standard one has been used to improve efficiency and reliability. The extruded profile is coated with a resistive layer of graphite having a typical surface resistivity between 0.2 and 0.4 $\mathrm{MOhm} /$ square. The tubes are assembled in modules and installed in 12 active layers of each sextant of the IFR detector. R\&D studies to choose the final design and Quality Control procedure adopted during the tube production will be briefly discussed. Finally the performances of installed LSTs into $2 / 3$ of IFR after 8 months of operations will be reported.
\end{abstract}

\section{The flux return geometry}

As originally built, the muon and $K_{L}^{0}$ detection system for BABAR consisted of 19 layers of resistive plate chambers (RPCs) interleaved with the flux return iron in the barrel region and 18 layers in the forward and backward endcaps. Each detector gap contained a single layer of RPCs based on a bakelite and linseed oil design. During initial BABAR operations, the temperature in the iron increased to as much as $30^{\circ} \mathrm{C}$. Since that time efficiencies have continued to decline at the rate of approximately $1.2 \%$ per month. Efforts to reverse or halt the underlying decline in efficiency have not been successful. All the chambers in the forward endcap were replaced in the summer and fall of 2002 with RPCs built with more stringent quality control (the upgrade project foresaw also the replacement of 5 active layers with $2.5 \mathrm{~cm}$ thick brass plates to improve the pion rejection. In December 2002 BABAR it has been decided to replace RPCs with limited streamer 
tubes (LSTs) for the barrel upgrade.

\section{The LST Concept}

A "standard" LST configuration [1] consists of a silver plated wire $100 \mu \mathrm{m}$ in diameter, located at the center of a cell of $9 \times 9 \mathrm{~mm}^{2}$ section. A plastic (PVC) extruded structure, or profile, contains 8 such cells, open on one side (Fig. 1). The profile is coated with a resistive layer of graphite, having a typical surface resistivity between 0.1 and $1 \mathrm{MOhm} /$ square. The profiles, coated with graphite and strung with wires, are inserted in plastic tubes ("sleeves") of matching dimensions for gas containment. The signals for the measurement of one coordinate can be read directly either from the wires or from external strip planes attached on both side of the sleeve.

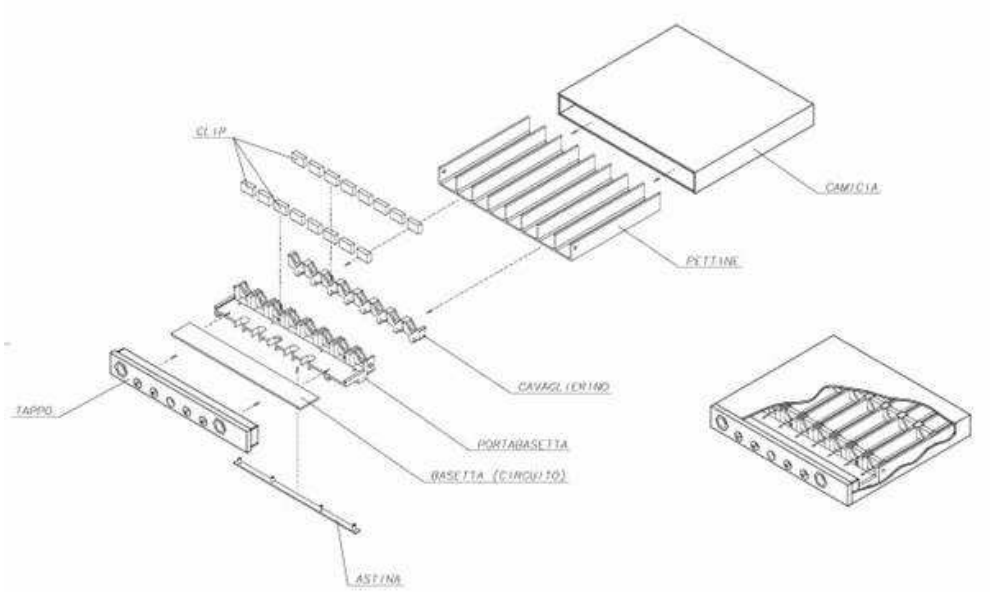

Figure 1. Schematic of the "standard" Limited Streamer Tube configuration.

\section{R\&D studies, Design and Performance}

More than one year of $\mathrm{R} \& \mathrm{D}$ studies have been done before choosing the final LST design. Our R\&D program has been concentrated on several critical issues like: selection of safe gas mixture, rate capability, wire surface quality and uniformity, aging test and performance of the prototypes. Detailed studies concerning all these issues can be found at [2].

The LST tubes are somewhat fragile mechanically so careful design, handling, and operation are of paramount importance in preventing failures. 
The "mortality" of the LSTs depends on the cell size, on the care and attention given during construction and installation, and on the strictness of the acceptance tests. Given these constraints to build a highly efficient detector means: reduce tube mortality and/or introduce redundancy to decrease its effect on detector efficiency; arrange tubes into modules that can be extracted and replaced and to minimize the dead space; feed each tube with one or more independent $\mathrm{HV}$ channels and finally locate $\mathrm{HV}$ distribution boxes and front end electronics on the outside of the detector. Such indications led us to a $15 \times 17 \mathrm{~mm}^{2}$ cell design (which is more reliable and efficient) where each tube is composed by 7 or 8 cells and assembled in modules. We use wire readout for the azimuthal coordinate, $\phi$, and strips plans for the $z$ coordinate (along the beam direction). In order to obtain high performances and to respect the safety requirements it has been chosen a ternary gas mixture of $\mathrm{Ar} / \mathrm{C}_{4} \mathrm{H}_{10} / \mathrm{CO}_{2}(3 / 8 / 89) \%$ [3].

\section{LST production and quality control}

The LST production has been commissioned to an external farm, the Pol. Hi. Tech (PHT), which also assembled all the prototype used for R\&D. We worked together with PHT to improve the cleanliness of the tubes and to setup a system of stringent quality (QC) control to guarantee the high performance of the product. The QC system was designed to check the quality of all mechanical components, the goodness and the resistivity of the graphite coating, the gas tightness of all assembled LSTs. Finally a set of electrical tests was applied to check the behavior and the performances of each LST under normal and critical electric (e.g. maximum allowed voltage) and physics (e.g. high particle rate simulated with a radioactive source) conditions. In order to be accepted for the installation into BaBar, each LST did have to satisfy all stringent criteria required for each test.

\section{Installation into BaBar}

The first 2 sextants were installed during summer 2004. While the remaining four will be installed in the 2006 shutdown. Installation began with the insertion into each gap of a strip plane covering an entire gap. These strips have been attached to the iron and supported by gravity. Then the modules were brought onto a supporting structure and inserted into the gap on top of the strip layer. The full installation and commissioning of the two sector took less than 2 months and has been completed on schedule. QC tests after installation certify the proper working of the apparatus. 


\section{Performances of the two LST-sextants}

BaBar experiment began RUN5 on March 2005 and after 8 months of operation LSTs are showing very good performances concerning number of dead channels, plateau curves, efficiency, muon identification and pion rejection. The main results will be described in the following.

Silent channels. Since the installation, the number of silent channels is quite constant during the time and it fluctuates around $0.2 \%$. There are a total of 2 dead $\Phi$-channels (which could be broken wire, broken HV cable or broken signal cables) over $1552(0.1 \%)$ and 7 dead Z-channels (which could be disconnected strips or broken signal cables) over $2284(0.3 \%)$.

Plateau curves. Plateau curves are monitored monthly and all LSTs show a plateau width varying between $300-600 \mathrm{~V}$ around the value of $5600 \mathrm{~V}$. In Fig.2 plateau curves of the 4 high voltage channels of a 7-cells LST are shown.

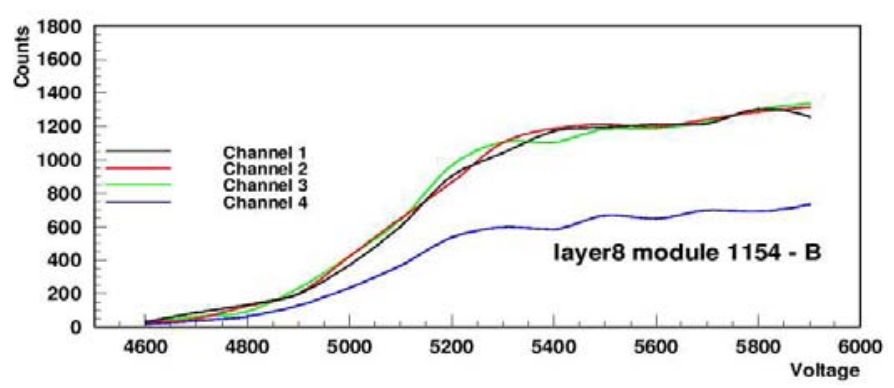

Figure 2. Plateau curves (counts versus voltage) of the 4 high voltage channels of a 7-cells LST; the firsts $3 \mathrm{HV}$ channels are are connected to 2 cells, while the $4^{\text {th }}$ channel are connected to one cell, then it shows half counts than the others.

Efficiency. The efficiency of installed LSTs is monitored daily using $\mu \mu$ pairs from colliding beams and monthly from cosmics rays. The calculated efficiency results to be constant around $90 \%$. The geometric efficiency is $92.5 \%$. The fluctuations of the efficiency are mostly related to the fluctuations on the number of silent channels, but no loss of efficiency for each single LST is detected. 
Muon ID and pion rejection. The Fig.3 shows the pion rejection as a function of the muon identification efficiency for high and low momentum. Comparing the results from LSTs and RPCs at different time it appears clear that LSTs are working better than RPCs ever did. LSTs introduced an excellent improvement in pion/muon discrimination.
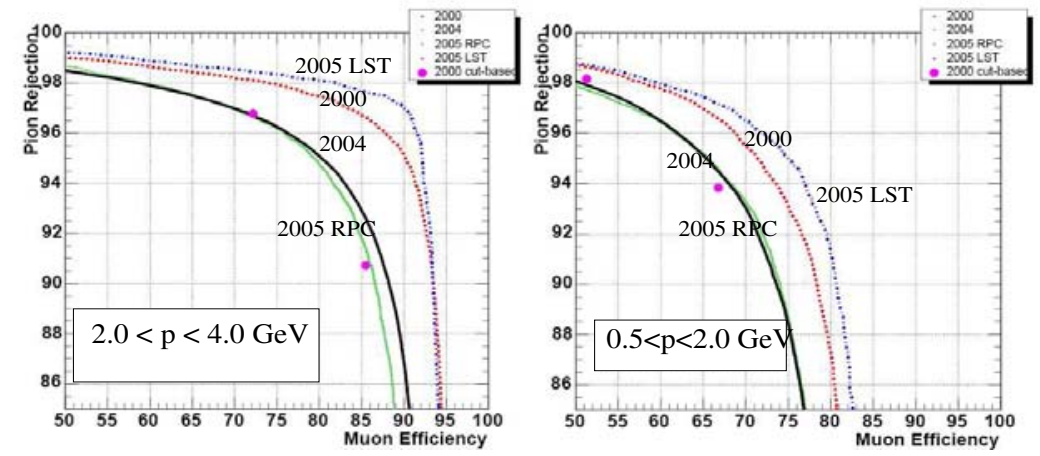

Figure 3. Pion rejection versus muon identification efficiency in IFR barrel region for RPCs during years 2000, 2004, 2005 and for LSTs during the year 2005.

Rate and current versus luminosity. There are a linear correlation between rate/current of the installed LSTs and the instantaneous luminosity. The corresponding slope shows that LSTs will work properly also at the early estimated instantaneous luminosity of $2 \cdot 10^{34} \mathrm{~cm}^{-2} \mathrm{~s}^{-1}$, only a firmware upgrade (already planned) of the high voltage power supply will be necessary to improve their performances.

The two sextants of barrel IFR instrumented with LSTs is working properly and it is showing good performances in terms of detection efficiency and muon identification.

\section{References}

1. G. Battistoni, E. Iarocci, M. M. Massai, G. Nicoletti and L. Trasatti, Operation of Limited Streamer Tubes, Nucl. Instrum. Meth. 164 (1979) 57.

2. BaBar LST Group, A barrel IFR Instrumented with Limited Streamer Tubes, (2003)

3. G.D. Alekseev, N. A. Kalinina, V. V. Karpukhin, D. M. Khazins and V. V. Kruglov, Investigation of Selfquenching Streamer discharge in a wire chamber, Nucl. Instrum. Meth. 177 (1980) 385. 